\title{
Ironing out the causes of B-cell dysfunction
}

New research published in Cell Host \& Microbe indicates a novel immunological role for the ironstorage protein ferritin in the B-cell defects that are characteristic of HIV-1 infection. Swingler and colleagues show that the HIV-1 accessory protein $\mathrm{Nef}$ induces the

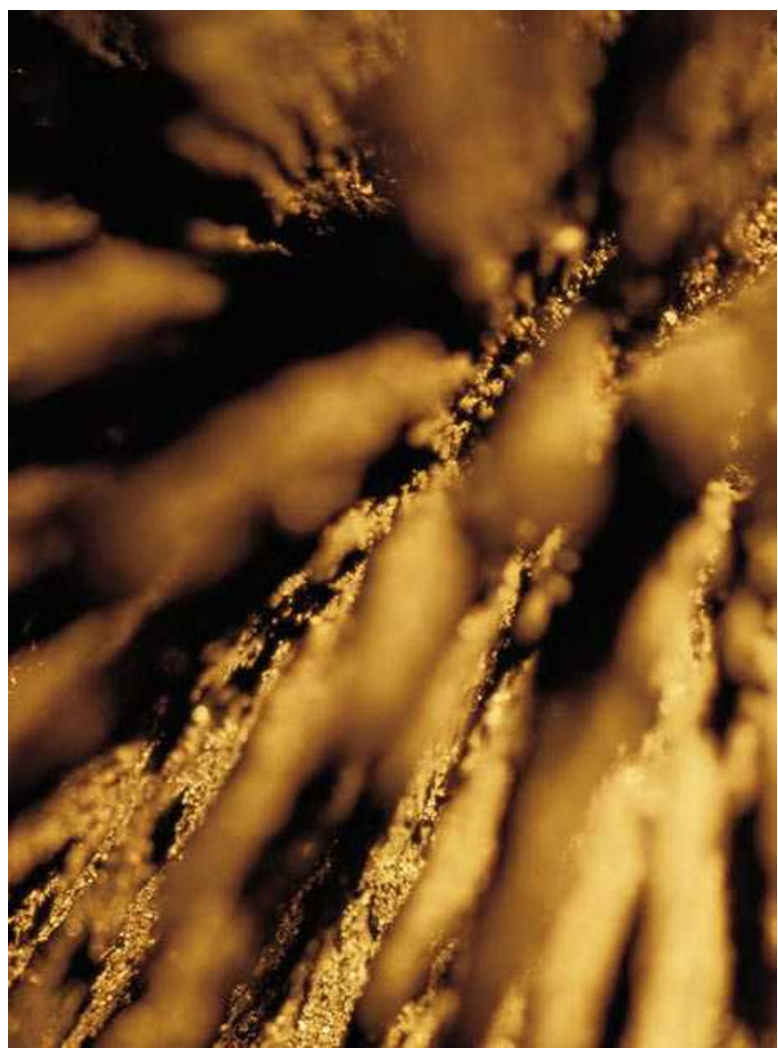

production of ferritin by infected macrophages, which in turn induces B-cell hyperactivation that could lead to B-cell exhaustion.

Supernatant from cultures of Nefexpressing or HIV-1-infected macrophages induced the proliferation of $\mathrm{B}$ cells in vitro, which was coincident with the upregulation of expression of activation markers such as CD38 and $\underline{\text { CD70 }}$, the plasma-cell marker CD138 (syndecan-1) and cell-surface IgM. Various methods were used to identify ferritin as the soluble factor produced by macrophages in response to Nef expression that was responsible for B-cell proliferation.

The Nef-induced production of ferritin by macrophages was shown to depend on the canonical nuclear factor- $\kappa \mathrm{B}(\mathrm{NF}-\kappa \mathrm{B})$ pathway. HIV-1-infected macrophages had increased expression of the NF- $\kappa \mathrm{B}$ p105 subunit and of the regulatory protein I $\mathrm{B} \alpha$ (inhibitor of NF- $\kappa \mathrm{B}, \alpha$ subunit), as well as phosphorylation of $I \kappa B \alpha$ on a regulatory serine that results in NF- $\mathrm{KB}$ activation. In an in vitro assay, Nef induced the activation of IкB kinase proteins, which are responsible for IKB phosphorylation and therefore NF- $\kappa B$ activation. Macrophages that expressed both $\mathrm{Nef}$ and a dominant-negative form of IкB $\alpha$ did not express ferritin above background levels.
B-cell proliferation was decreased to background levels when ferritin was immunodepleted from macrophage supernatants, but not when Nef was immunodepleted, which indicates that there is no direct effect of extracellular Nef on B-cell activation. In confirmation of the ferritin-mediated effects, purified human liver ferritin induced B-cell proliferation and expression of CD38, CD70 and CD138, as well as a dose-dependent increase in the production of IgA, IgG and IgM.

In a cohort of $83 \mathrm{HIV}$-1-infected individuals, there was a statistically significant correlation between plasma viral RNA load, plasma ferritin concentration and plasma levels of $\operatorname{IgA}$, IgG and IgM. So, in agreement with the in vitro findings, the extent of viral replication in vivo determines the level of ferritin production, which in turn determines B-cell hyperactivation.

The authors therefore suggest that the Nef-induced production of ferritin by HIV-1-infected macrophages is one of several mechanisms that are responsible for B-cell dysfunction in HIV/AIDS, which impairs the development and maintenance of a normal humoral immune response. Kirsty Minton

ORIGINAL RESEARCH PAPER Swingler, S. et al. Evidence for a pathogenic determinant in HIV-1 Nef involved in B-cell dysfunction in HIV/AIDS. Cell Host Microbe 4, 63-76 (2008) 